\section{JUNCTION LINE FILTERS}

T WO articles on these filters by F. A. Hinshaw and J. O. Israel respectively (Bell Lab. Rec., 20, No. 9, May 1942) describe their design and applications in communication circuits. Open-wire lines brought into repeater stations or toll offices located in densely populated areas are usually terminated at the outskirts of the area, and the circuits are brought to the office through cables. These cables are usually loaded so as to improve the impedance match between the open-wire line and the cable and to reduce the loss in the cable. At repeater stations and terminals the separation of the carrier and voice circuits is ordinarily accomplished by filters at the office end of the cable. It is not feasible to use the office filters to separate the carrier and voice circuits at the terminal pole to avoid the necessity of carrier loading. Moreover, difficulties would be encountered in obtaining a suitable balance at the voice repeater because of the imperfect impedance match of filter and cable, and of the length of cable between filter and repeater, which would be different for each installation.

These difficulties have been overcome by the development of the junction line filter and its associated networks, the principal duty of which is to separate carrier and voice currents. This separation is accomplished by two single-section filters, a low-pass and a high-pass. As soon as these filters are introduced into the line, however, several other factors become important, the most outstanding of which are longitudinal balance, reflection coefficient, and modulation. Two junction filters are available. In one, the low-frequency channel is wide enough for a 5 kc. programme circuit, while the high-frequency channel is suitable for open-wire carrier telephone systems operating at frequencies as low as $6 \cdot 5 \mathrm{kc}$. In the other, the low-frequency channel is wide enough for voice frequencies up to $3 \mathrm{kc}$., while the high-frequency channel passes frequencies as low as $4.8 \mathrm{kc}$.

By the use of these junction filters, carrier loading of the longer entrance cable is unnecessary; the voice frequencies are brought in over a voice-loaded pair, and the carrier frequencies over a non-loaded pair. Certain additional equipment is also required. An impedance-matching network on the cable side of the high-pass filter corrects the impedance mismatch that would otherwise exist at carrier frequencies between the filter and non-loaded cable pair. At the office end of the carrier pair a transformer network transforms the relatively low impedance of the non-loaded cable pair to the office impedance. Since with this arrangement the office filters are no longer connected in parallel, compensating networks are required to simulate the impedance effect of one filter on the other.

The insertion of the filter at the terminal pole, however, introduces an impedance irregularity in the voice frequency pair and upsets the balance at the hybrid coil. To maintain a suitable balance, therefore, the effect of the filter and the length of cable must be duplicated in the balancing network. Where loaded entrance cable is connected directly to the open-wire line, the balancing network includes a section to simulate the low-pass filter, a section to simulate the composite set, and a two-terminal network to simulate the impedance of the open-wire line or loaded entrance cable. Where the junction line filter is employed, however, the network must include other elements to simulate both the filter and the particular length of entrance cable involved.

For simulating the transmission characteristics of the entrance cable, which may be of different length for each installation, a series of cable-simulating units has been provided. Three types of simulating networks have been developed and with a suitable combination of them and a suitable amount of building-out, any length of entrance cable can be simulated. Terminal sections are built up in a similar manner to simulate the characteristics of the terminal section.

The ability of the junction filter to operate out-ofdoors under all kinds of weather conditions and its comparatively low cost make it an economical substitute for carrier loading on the longer entrance cables. While the junction filter gives transmission results sufficiently satisfactory to justify its use, it does not provide as good overall impedance matching as carrier loading, and it increases rather than decreases entrance cable losses.

\section{'TACK' AND STICKY MATERIALS}

A $\mathrm{N}$ informal discussion on the subject of 'tack' $A$ and sticky materials, arranged jointly by the British Rheologists' Club and the Printing and Allied Trades Research Association, was held at St. Bride Institute, Fleet Street, London, on June 10. Dr. G. L. Riddell presided.

In his introductory remarks, Dr. V. G. W. Harrison said that the purpose of the meeting was to bring together those interested in 'sticky' materials. It is still uncertain what physical properties produce 'stickiness' and it was hoped that as a result of the meeting ideas would be clarified and perhaps some preliminary investigations could be started. Those present represented a wide range of interests. and it was generally felt that an informal meeting of this kind was long overdue.

Mr. C. C. Mill opened the discussion. 'Tack', he said, is a common term in the printing and allied trades. It means, in a general way, 'stickiness', but it has no precise definition and is usually estimated by a 'finger test'. A certain amount of 'tack' is considered essential in printing ink, and it is thus desirable to have some means of defining and measuring this property. Various attempts have already been made. Recently Green (Ind. and Eng. Chem. Anal. Edit., 13, 632; 1941) has described a method which attempts to reproduce the finger test. $\mathrm{He}$ arrives at an equation in which the time taken to move a mechanical 'finger' a fixed distance is a function of the geometry of the 'finger' system and the viscosity of the material tested.

If Green was really measuring 'tack', Mr. Mill continued, then this property is identical with viscosity, and all liquids of the same viscosity should have the same 'tack', which is contrary to the usual belief. It seems probable that Green over-simplified the problem. In addition to the stress required to maintain viscous flow between the separating surfaces, a further force is required to break the film. This force is dependent on the cohesive and adhesive properties of the liquid, and thus varies from one liquid to another. Another possible factor causing 'tack' is suggested by Maxwell's conception of 'relaxation time'. With an ideal liquid the stress produced by a given strain is rapidly dissipated, but in 